\title{
Skin bacterial flora as a potential risk factor predisposing to late bacterial infection after cross-linked hyaluronic acid gel augmentation
}

This article was published in the following Dove Press journal: Infection and Drug Resistance

\author{
Irina Netsvyetayeva' \\ Wojciech Marusza ${ }^{2}$ \\ Romuald Olszanski ${ }^{3}$ \\ Kamila Szyller ${ }^{2}$ \\ Aneta Krolak-Ulinska ${ }^{2}$ \\ Ewa Swoboda-Kopec' \\ Janusz Sierdzinski ${ }^{4}$ \\ Zachary Szymonski ${ }^{5}$ \\ Grazyna Mlynarczyk' \\ 'Department of Microbiology, \\ Medical University of Warsaw, \\ Poland; ${ }^{2}$ Academy of Face Sculpturing, \\ Warsaw, Poland; ${ }^{3}$ Military Institute \\ of Health Services, Warsaw, Poland; \\ ${ }^{4}$ Department of Medical Informatics \\ and Telemedicine, Medical University \\ of Warsaw, Poland; ${ }^{5}$ Department of \\ Zoology, Magdalen College, University \\ of Oxford, Oxford, UK
}

Correspondence: Wojciech Marusza Academy of Face Sculpturing, ul. Jana Kazimierza IIB, 00-I50 Warsaw, Poland Email drmarusza@drmarusza.pl
Introduction: Cross-linked hyaluronic acid (HA) gel is widely used in esthetic medicine. Late bacterial infection (LBI) is a rare, but severe complication after HA augmentation. The aim of this study was to determine whether patients who underwent the HA injection procedure and developed LBI had qualitatively different bacterial flora on the skin compared to patients who underwent the procedure without any complications.

Methods: The study group comprised 10 previously healthy women with recently diagnosed, untreated LBI after HA augmentation. The control group comprised 17 healthy women who had a similar amount of HA injected with no complications. To assess the difference between the two groups, their skin flora was cultured from nasal swabs, both before and after antibiotic treatment in the study group.

Results: A significant increase in the incidence of Staphylococcus epidermidis was detected in the control group $(P=0.000)$ compared to the study group. The study group showed a significantly higher incidence of Staphylococcus aureus $(P=0.005)$, Klebsiella pneumoniae $(P=0.006)$, Klebsiella oxytoca $(P=0.048)$, and Staphylococcus haemolyticus $(P=0.048)$ compared to the control group.

Conclusion: The bacterial flora on the skin differed in patients with LBI from the control group. The control group's bacterial skin flora was dominated by $S$. epidermidis. Patients with LBI had a bacterial skin flora dominated by potentially pathogenic bacteria.

Keywords: hyaluronic acid, late bacterial infection, bacterial biofilm, skin bacterial flora, S. epidermidis, S. aureus, Klebsiella spp.

\section{Introduction}

Late bacterial infection (LBI) after administration of cross-linked hyaluronic acid (HA) gel during an esthetic procedure on the face is a complication that is difficult to treat and sometimes not reported by doctors. Up to now, the true incidence of LBI in patients undertaking HA treatment was unknown. Literature shows that this complication has an occurrence rate ranging from $0.01 \%$ to $0.1 \% .^{1,2}$ Unfortunately, there are few publications on, as well as little knowledge about, the causes of LBI. HA gel is currently one of the safest biodegradable materials used in esthetic medicine. Uses for its non-cross-linked form include mesotherapy to moisturize and improve skin condition, while the cross-linked form is often subcutaneously injected as a filler. ${ }^{3}$ Compounds containing HA are the most commonly used biodegradable fillers in Europe and the USA. Their effect lasts from 6 to 18 months, depending upon the type and degree of cross-linking in the compound, along with 
the concentration and size of the constituent particles. HA compounds contain polymerized dimers of $\mathrm{N}$-acetylglucosamine and D-glucuronic acid, which are characterized by differences in their linkages, density, and uniformity. These characteristics have an enormous influence on the effects of different HA compounds. Increased cross-linking and higher concentration alter the viscosity and elasticity of the compound, and increase its resistance to degradation by naturally occurring hyaluronidase, thus extending the duration of the effect. ${ }^{4}$ Most of the complications occurring after the application of cross-linked HA are the result of the following errors: improper disinfection of the skin during treatment, application of the compound in an incorrect location, usage of an excessive volume, or incorrect injection technique. ${ }^{5}$ It was recently observed that an increase in complications often arises after this procedure due to hypersensitivity to components of the HA product. It must be emphasized that foreign body granuloma formation, as well as infections at the site of application, are common side effects of any treatment passing through the skin. ${ }^{6,7}$ The time frame in which complications occur ranges from a few days to possibly several months after treatment. Suspected causes include impurities in HA formed during its production, HA degradation triggering allergic reactions, as well as bacterial contamination within HA, which can cause local inflammation. ${ }^{8}$ Complications of bacterial origin can include widespread skin and connective tissue infections, erysipelas, pustules, abscesses, and inflammatory nodules. Microorganisms causing erysipelas and abscesses are usually Staphylococcus aureus or Streptococcus pyogenes. These complications usually arise early, up to 2 weeks after treatment, while bacterial abscess is a rare complication that may arise anytime from 1 week to a few years after the treatment, and progresses in a rapid and aggressive mode. It has a tendency to recur and may require surgical intervention. Lastly, inflammatory nodule complications may be linked to the growth of bacteria in the form of a biofilm. ${ }^{9}$ The suspected causes of bacterial biofilm infection (BBI) are mostly Staphylococcus epidermidis and $S$. aureus. ${ }^{1}$ It has been reported that even very minor contamination of HA by bacteria is sufficient for the development of a biofilm. ${ }^{9}$ Typically, a culture of the pus from an inflammatory nodule tests negative for the presence of any bacteria. Thus, these nodules had long been considered to be an allergic reaction or response to the filler material as a foreign body. ${ }^{4}$ The allergic response to $\mathrm{HA}$ is suggested to be a type IV immunological reaction.
Published articles are indicative that this allergic response is unusual and rare and are suggestive that BBI-LBI has a more frequent occurrence. ${ }^{1,2,9}$ The characteristics of the mentioned allergic reactions are completely different than those observed in patients with BBI-LBI symptoms. ${ }^{10}$ Additionally, if there is an allergic reaction, it always occurs at every site of HA, although BBI-LBI may occur at one of many places following HA injection. Patients with an allergic reaction have a normal erythrocyte sedimentation rate (ESR) and patients with BBI-LBI generally have a raised ESR. Inflammatory nodules can be diagnosed by noticing symptoms including redness, swelling, hardness, tenderness, and purulent discharge in conjunction with raised ESR, which is a nonspecific measure of inflammation in many conditions: bacterial and/or viral infection, autoimmune diseases, dysproteinemia, and cancers. ${ }^{6,11}$ Fluorescence in situ hybridization can be used to determine the bacterial cause of an inflammatory nodule BBI. ${ }^{12}$. Patients, who are looking to undergo an esthetic procedure, should be assessed through a medical interview to avoid posttreatment complications. ${ }^{4}$ The factors listed in the literature that could contribute to increasing risk of bacterial complication on the patient's face are preexisting skin infections/inflammation or injury, periodontal infections, sinusitis, and inadequate skin disinfection. ${ }^{13}$ However, there is a lack of data concerning the importance of normal bacterial skin flora on the subsequent occurrence of postinjection HA bacterial complications.

\section{Aim of the study}

The aim of this study was to answer the question whether there is a link between disturbances of normal bacterial skin flora before the procedure and post-procedure complications, such as bacterial inflammatory nodules formed on cross-linked HA-LBI, which is difficult to treat because of resistance to standard antibiotics.

\section{Materials and methods}

The study was conducted at the Akademia Rzeźbienia Twarzy (Academy of Face Sculpturing [AFS], Warsaw, Poland) in the years 2012-2015. The individuals participating in the study were healthy women between the ages of 31 and 71 years (mean age 44.8 years).

All participants provided written informed consent. The research was approved by the Ethical Committee of the Department of Microbiology of Warsaw Medical University. 


\section{Enrollment criteria for participation in the study}

The enrollment criteria for patient participation in the study were as follows: 1) absence of skin diseases and local inflammatory sites, including periodontitis, sinusitis, systemic infections, autoimmune diseases, immunosuppression, and hormonal dysfunction requiring therapy; 2) having undergone an esthetic procedure with the use of 1-3 $\mathrm{mL}$ cross-linked HA gel containing 20-26 mg/mL stabilized cross-linked HA, which was injected into facial tissue. HA was produced by bacterial fermentation, which is legally permitted for use in esthetic medicine in Poland. The patients were treated with HA from various manufacturers; 3 ) absence of antibiotics for 1 month prior to sample collection; 4) a signed consent form.

\section{Study group}

The group consisted of 10 healthy women who had a procedure involving HA injection and fulfilled the criteria of 1 month absence of antibiotics prior to the first microbiological sample being taken. The women were 31-56 years of age (mean age 43.8 years) and were referred by various esthetic doctors in Poland. They presented to the AFS with symptoms of an infection, LBI, as a complication in at least one site of injection of cross-linked HA. The time frame from the initial administration of HA to the appearance of LBI was between 1 and 18 months (mean time 5.2 months). The patients had been treated with HA from various manufacturers.

\section{Enrollment criteria for the study group}

The enrollment criteria for inclusion in the study group were local symptoms of bacterial infection (LBI) - redness, swelling, hardness, tenderness, pus at the site of cross-linked HA gel augmentation, which appeared between 1 and 18 months after HA gel augmentation, and increased ESR, above $10 \mathrm{~mm} /$ hour.

\section{Criteria for recovery}

The criteria for recovery were absence of general and local symptoms of bacterial infection, such as redness, swelling, hardness, tenderness, pus, and raised ESR, and an asymptomatic state for at least 30 days.

\section{Control group}

The control group consisted of 17 healthy women aged 31-71 years (mean age 45.8 years), who were regular clients of the AFS, and who did not develop any complications after at least three repeated treatments with HA injections within 24 months.

\section{Microbiological diagnosis Sample collection}

Swabs were taken from both nostrils at the same time using triple rotation of a transport swab (Oxoid, Basingstoke, UK). Samples were taken four times from each individual in the study group and three times from each individual in the control group. Sample " 0 " was taken only from the study group on the day when the individual reported to the clinic with inflammation at the site of cross-linked HA gel augmentation. Sample "1" was taken in the study group within 4-8 weeks after recovery from inflammation at the site of crosslinked HA gel augmentation, on the day of the visit where the patient had a normal ESR and C-reactive protein (CRP) level. In the control group, a sample was taken on the day of enrollment, where the patient had no signs of inflammation at the site of cross-linked HA gel augmentation and a normal ESR and CRP level. Sample "2" was taken in both groups 4-8 weeks after taking sample " 1 " and fulfilled the requirements of sample 1. Sample "3" was taken in both groups 4-8 weeks after taking sample " 2 " and fulfilled the requirements of sample 1. Additionally, samples were taken in the study group from the pus and from the inflammatory changes at the site of cross-linked HA gel augmentation, on the day of enrollment to the study, onto transport swabs (Oxoid). None of the patients agreed to a diagnostic biopsy.

\section{Microorganism isolation}

The collected samples were cultured at the Department of Clinical Microbiology at Warsaw Medical University within 36 hours of being taken. The following microbiological substrates were used to culture the microorganisms: Columbia agar with 5\% sheep blood, mannitol salt agar, MacConkey agar, chocolate agar (chocolate Haemophilus agar), as well as Schaedler agar (all from BioMaxima SA, Centrum Mikrobiologii Emapol, Poland). The cultures were incubated for $24-48$ hours at $35^{\circ} \mathrm{C}$ under microaerophilic aerobic conditions for bacteria on chocolate agar medium and anaerobic conditions for bacteria on the Schaedler agar medium. After incubation, the colonies of microbes were isolated and recultured to enable identification.

\section{Identification of bacterial species}

The bacterial isolates were identified by matrix-assisted laser desorption/ionization-time of flight mass spectroscopy, using an IVD MALDI BIOTYPER system (Bruker Optik 
GmbH, Ettlingen, Germany), according to the manufacturer's instruction.

\section{Methicillin-resistant S. aureus (MRSA) diagnostics}

Susceptibility to methicillin was determined using the disc diffusion antibiotic sensitivity testing method, also known as Kirby-Bauer antibiotic testing, according to the recommendations from EUCAST 2015. A Mueller-Hinton agar (Graso Biotech, Starogard Gdanski, Poland) and discs with cefoxitin (30 $\mu \mathrm{g}$; Oxoid) were used.

\section{Carriers}

Persistent carriers of bacterial species were considered to be individuals in whom three samples were positive; transient carriers were considered to be individuals in whom one or two samples were positive.

\section{Statistical analysis}

Statistica 12.0 package was used to evaluate the results. Subsequently, further analysis of the results was carried out. Nonparametric tests for unlinked characters were performed using chi-square test and Fisher's test, and for linked characters using McNemar's tests. The value of $P<0.05$ was set for the results to be considered significant.

\section{Results}

No bacterial growth was observed in any of the samples taken from the pus and from the inflammatory changes at the site of cross-linked HA gel augmentation in the study group. In total, 91 cultures from nasal swabs were examined. Forty came from the study group (four from each of the 10 individuals). The other 51 swabs (three from each of the 17 individuals) came from the control group, and were taken from both nostrils in intervals of 4-8 weeks. The results of the research can be found in the data tables. Table 1 shows the results obtained from the study group, while Table 2 shows results from the control group. Analyzing the samples taken at the first report of symptoms from the 10 individuals in the study group (ie, sample “0”), S. epidermidis was found in six patients (60\%); S. aureus in four (40\%); Corynebacterium accolens in three (30\%); Klebsiella pneumoniae, Klebsiella oxytoca, Staphylococcus warneri, and Propionibacterium acnes in two (20\%); and Citrobacter koseri, Streptococcus oralis, and Staphylococcus haemolyticus in one patient $(10 \%)$. In the 30 samples taken three times from each of 10 individuals in the study group, in 4-8 week intervals after curing the inflammation, $S$. epidermidis was found in 12 samples (40\%); S. aureus in 15 (50\%); K. pneumoniae in five $(16.7 \%) ;$ K. oxytoca in three $(10 \%)$; S. haemolyticus in three (10\%); Corynebacterium tuberculostearicum in two (6.7\%); and Acinetobacter ursingii, Pseudomonas oryzihabitans, and $S$. warneri in one (3.3\%). C. accolens and $P$. acnes were not present. In the 51 samples taken from each of the 17 individuals from the control group, three times in 4-8 week intervals, the presence of $S$. epidermidis was confirmed in all 51 samples (100\%); S. aureus in nine (17.6\%); P. acnes in five (9.8\%); C. accolens in four (7.8\%); $S$. warneri in three (5.9\%); and A. ursingii, C. tuberculostearicum, and P. oryzihabitans in two. In the samples taken from the control group, K. pneumoniae, K. oxytoca, and $S$. haemolyticus were not present. These results are presented in Figure 1. The occurrence of Gram-negative bacteria of the family Enterobacteriaceae was found in two of 51 samples (3.9\%) from the control group. These were isolated cases of Enterobacter cloacae and Pantoea spp. from two out of $17(11.8 \%)$ individuals. In the study group, the occurrence of the family Enterobacteriaceae was found in 16 out of 40 (40\%) samples taken from seven out of $10(70 \%)$ patients. Persistent carriage of $S$. aureus was found in four patients from the study group (40\%), as well as in one in the control group $(5.9 \%)$. For four individuals $(40 \%)$ in the study group and nine individuals (52.9\%) in the control group, S. aureus bacteria was not found. Transient occurrence of $S$. aureus was found in two individuals (20\%) in the study group, and in seven $(41.2 \%)$ in the control group. MRSA was found in two samples taken from one patient in the study group, when samples were collected after the treatment of bacterial complication. Furthermore, the occurrence of $K$. pneumoniae was found in 3 and 4 samples in two patients, and $K$. oxytoca in 4 samples in one patient from the study group. Statistically significant differences in the occurrence of $S$. epidermidis ( $P=0.000)$, S. aureus $(P=0.005), K$. pneumoniae $(P=0.006)$, K. oxytoca $(P=0.048)$, and $S$. haemolyticus $(P=0.048)$ were found between cultures from the study and control groups. These were cultured from samples taken three times in the control group and taken three times after curing the inflammation in the study group. In the study group, no significant differences were found between sample 0 and samples $1-3$.

\section{Discussion}

In order to answer questions regarding LBI in healthy individuals following a procedure involving HA injection, we decided to collect data on patients with this complication across Poland over a period of 3 years. Treatments using HA were administered by various esthetic doctors in Poland. In every case, it was the first encounter with such a complication in the doctor's practice. During this period, we had 26 patients 


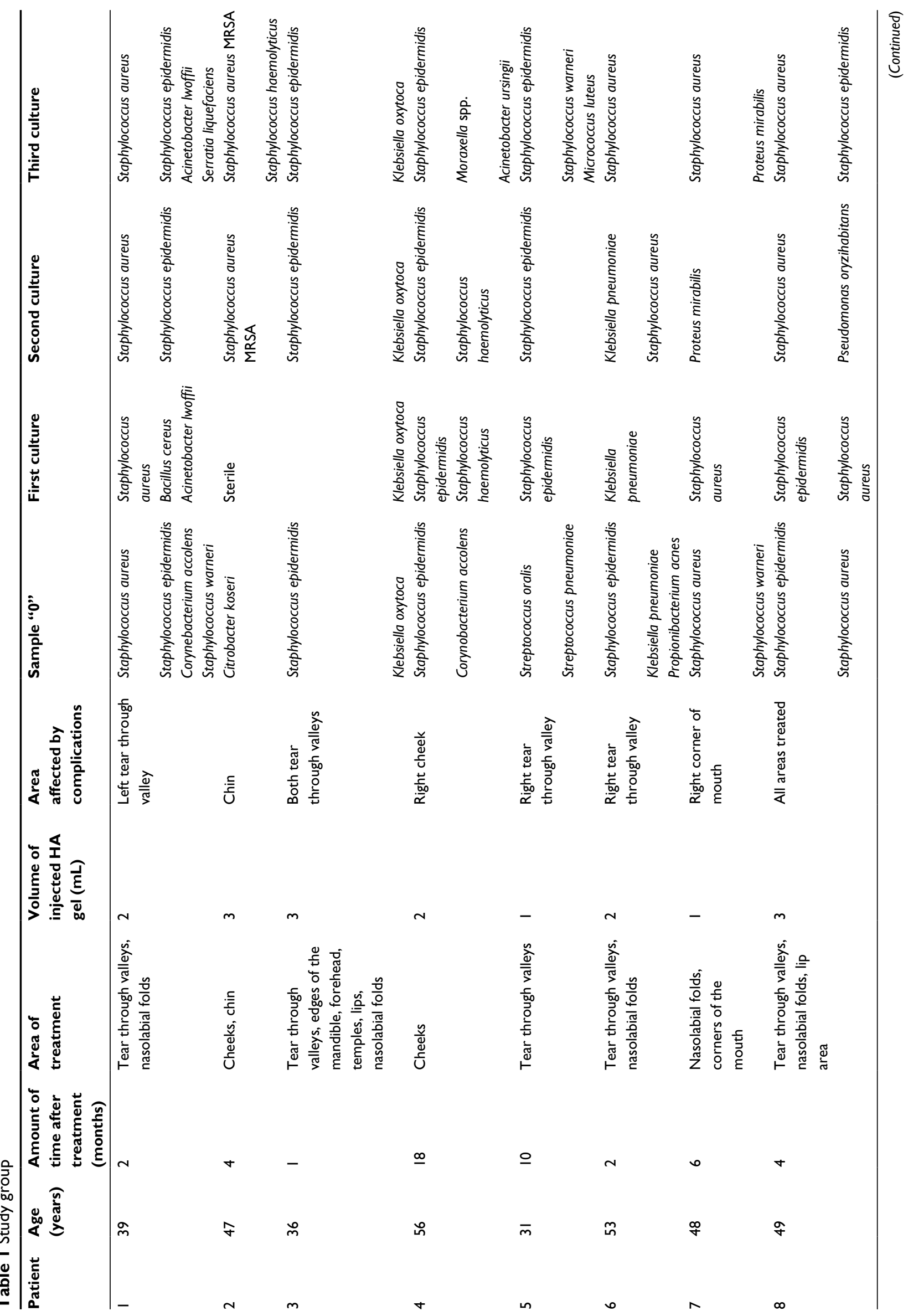


with LBI following a procedure involving HA, out of whom we selected a group of 10 patients fulfilling the study criteria. In all these patients, inflammation developed at the site of HA gel augmentation during a period of 1-18 months after the esthetic procedure (mean: about 5 months). According to the classification published by Funt and Pavicic, the time that had elapsed since the development of the complication, along with inflammatory response indicators like ESR elevation in the study group, points toward the late occurring nature of the infection. Thus, this particular complication is linked to LBI - probable bacterial biofilm growth on the surface of the HA gel. An additional argument for BBI in favor of this conclusion is the negative result of cultures made from samples of the pus at the site of infection. ${ }^{4}$ In eight of the 10 chosen patients, the LBI complication was present only in one or two of the multiple sites of HA injection. In two of the 10 chosen patients, the complication was present at each site of injection (Table 1). We were not able to confirm BBI due to the lack of consent from the patients for taking biopsies. It is now generally accepted that LBI that is formed in the HA implant site is associated with biofilm formation. ${ }^{14,15}$ The cross-linked HA acts like an implant, and therefore can be used to explain causes of biofilm formation. Incidences of LBI, probable BBI, shown in the medical literature are inflammatory reactions at the injection site after $>1$ month from the administration of cross-linked HA, to a lasting effect of HA in the subcutaneous tissue for $\sim 2$ years. ${ }^{4,6,9,12}$ Features of the infection are swelling, hardening, reddening, tenderness, and pus discharge. Additionally, in all patients from the study group, we confirmed the occurrence of raised ESR, which is a test widely used in general medicine, confirming inflammation mainly caused by bacteria. ESR may also be increased in autoimmune diseases, dysproteinemia, cancers, or viral infections. ${ }^{11}$ All causes of raised ESR other than local bacterial infections were excluded in the examined patients. In our tests, every patient from the study group had a raised ESR and clear symptoms of inflammatory nodules: reddening, swelling, hardening, pain, and pus discharge at the site of HA injection. The relevance of proper clinical diagnosis was confirmed by the fact that all the patients were treated successfully with dual-antibiotic therapy in conjunction with the hyaluronidase enzyme, which by dissolving the HA gel removed the platform on which the biofilm was formed. Once the structure of the biofilm is destroyed, antibiotics can gain access to the bacterial cells. The indicator for the recovery of the patients was the disappearance of all inflammation symptoms and normalization of ESR. Local delayed infection after cross-linked HA gel augmentation could be linked to 
Table 2 Control Group

\begin{tabular}{|c|c|c|c|c|}
\hline Patient & Age (years) & First culture & Second culture & Third culture \\
\hline \multirow[t]{2}{*}{$\mathrm{I}$} & 47 & Staphylococcus aureus & Staphylococcus aureus & Staphylococcus aureus \\
\hline & & Staphylococcus epidermidis & Staphylococcus epidermidis & Staphylococcus epidermidis \\
\hline \multirow[t]{2}{*}{2} & 51 & Staphylococcus epidermidis & Staphylococcus epidermidis & Staphylococcus epidermidis \\
\hline & & Corynebacterium accolens & Corynebacterium accolens & \\
\hline \multirow[t]{2}{*}{3} & 71 & Staphylococcus epidermidis & Staphylococcus epidermidis & Staphylococcus epidermidis \\
\hline & & & & Staphylococcus aureus \\
\hline \multirow[t]{3}{*}{4} & 56 & Staphylococcus epidermidis & Staphylococcus epidermidis & Staphylococcus epidermidis \\
\hline & & Acinetobacter ursigni & Staphylococcus aureus & Acinetobacter ursigni \\
\hline & & Brevibacterium celere & Pseudomonas oryzihabitans & \\
\hline \multirow[t]{2}{*}{5} & 69 & Staphylococcus epidermidis & Staphylococcus epidermidis & Staphylococcus epidermidis \\
\hline & & & & Staphylococcus intermitidis \\
\hline \multirow[t]{3}{*}{6} & 47 & Staphylococcus epidermidis & Staphylococcus epidermidis & Staphylococcus epidermidis \\
\hline & & Propionibacterium acnes & & Propionibacterium spp. \\
\hline & & & & Corynebacterium accolens \\
\hline \multirow[t]{2}{*}{7} & 31 & Staphylococcus epidermidis & Staphylococcus epidermidis & Staphylococcus epidermidis \\
\hline & & Corynebacterium tuberculostearicum & & Propionibacterium acnes \\
\hline 8 & 34 & Staphylococcus epidermidis & Staphylococcus epidermidis & Staphylococcus epidermidis \\
\hline \multirow[t]{2}{*}{9} & 33 & Staphylococcus epidermidis & Staphylococcus epidermidis & Staphylococcus epidermidis \\
\hline & & Enterobacter cloacae & Staphylococcus aureus & Corynebacterium amycolatum \\
\hline \multirow[t]{2}{*}{10} & 36 & Staphylococcus epidermidis & Staphylococcus epidermidis & Staphylococcus epidermidis \\
\hline & & & Staphylococcus warneri & \\
\hline \multirow[t]{2}{*}{ II } & 50 & Staphylococcus epidermidis & Staphylococcus epidermidis & Staphylococcus epidermidis \\
\hline & & & Corynebacterium accolens & Pantoea sp. \\
\hline \multirow[t]{2}{*}{12} & 61 & Staphylococcus epidermidis & Staphylococcus epidermidis & Staphylococcus epidermidis \\
\hline & & & Staphylococcus aureus & \\
\hline \multirow[t]{3}{*}{13} & 30 & Staphylococcus epidermidis & Staphylococcus epidermidis & Staphylococcus epidermidis \\
\hline & & Micrococcus luteus & Pseudomonas oryzihabitans & Propionibacterium acnes \\
\hline & & Staphylococcus aureus & & \\
\hline \multirow[t]{2}{*}{14} & 30 & Staphylococcus epidermidis & Staphylococcus epidermidis & Staphylococcus epidermidis \\
\hline & & Staphylococcus aureus & & Staphylococcus aureus \\
\hline \multirow[t]{3}{*}{15} & 34 & Staphylococcus epidermidis & Staphylococcus epidermidis & Staphylococcus epidermidis \\
\hline & & Staphylococcus aureus & Propionibacterium acnes & Staphylococcus warneri \\
\hline & & & Staphylococcus warneri & \\
\hline \multirow[t]{2}{*}{16} & 51 & Staphylococcus epidermidis & Staphylococcus epidermidis & Staphylococcus epidermidis \\
\hline & & & & Propinibacterium Acnes \\
\hline \multirow[t]{2}{*}{17} & 48 & Staphylococcus epidermidis & Staphylococcus epidermidis & Staphylococcus epidermidis \\
\hline & & Corynebacterium tuberculostearicum & & \\
\hline
\end{tabular}

risk factors related to the patient's condition. HA gel augmentation requires the absence of active skin infections, active local and general infections, and active autoimmune diseases in the patient. Other conditions that must be considered are atopic allergic dermatitis, sensitive skin syndrome, seborrheic dermatitis, noninfectious gastrointestinal diseases, organ transplant, or thyroid dysfunction. ${ }^{5}$ Only patients showing none of the above conditions qualified for participation in the study. It has been shown by many researchers that symbiotic microorganisms inhabiting the skin act as a protective barrier to infection and play a key role in determining the cellular response to a pathogen. ${ }^{16}$ The aim of this study was to compare the bacteria cultured from the nostrils of individuals who had LBI with those who did not experience any complications after at least three crosslinked HA augmentations. Nasal swabs were used to quali- tatively evaluate the composition of the physiological flora. Nostrils have relatively stable bacterial flora similar to facial skin. ${ }^{17}$ Another point in favor of this approach was that a culture from nasal swabs is regularly used to determine the presence of $S$. aureus, which is a factor that increases the risk of developing $S$. aureus infections. ${ }^{18}$ Sample "0" was taken to determine the bacterial flora in the individual, bearing in mind that the presence of pathogens may led to the inflammation. The following samples, taken three times subsequently, were meant to determine the qualitative composition of the bacterial flora in the recovery period for individuals who had LBI in the past. In addition, these samples were also used to determine carriers of $S$. aureus and other species of bacteria. Interestingly, there was a lack of significant differences between the qualitative composition of bacterial flora in individuals from the study group during the ongoing 


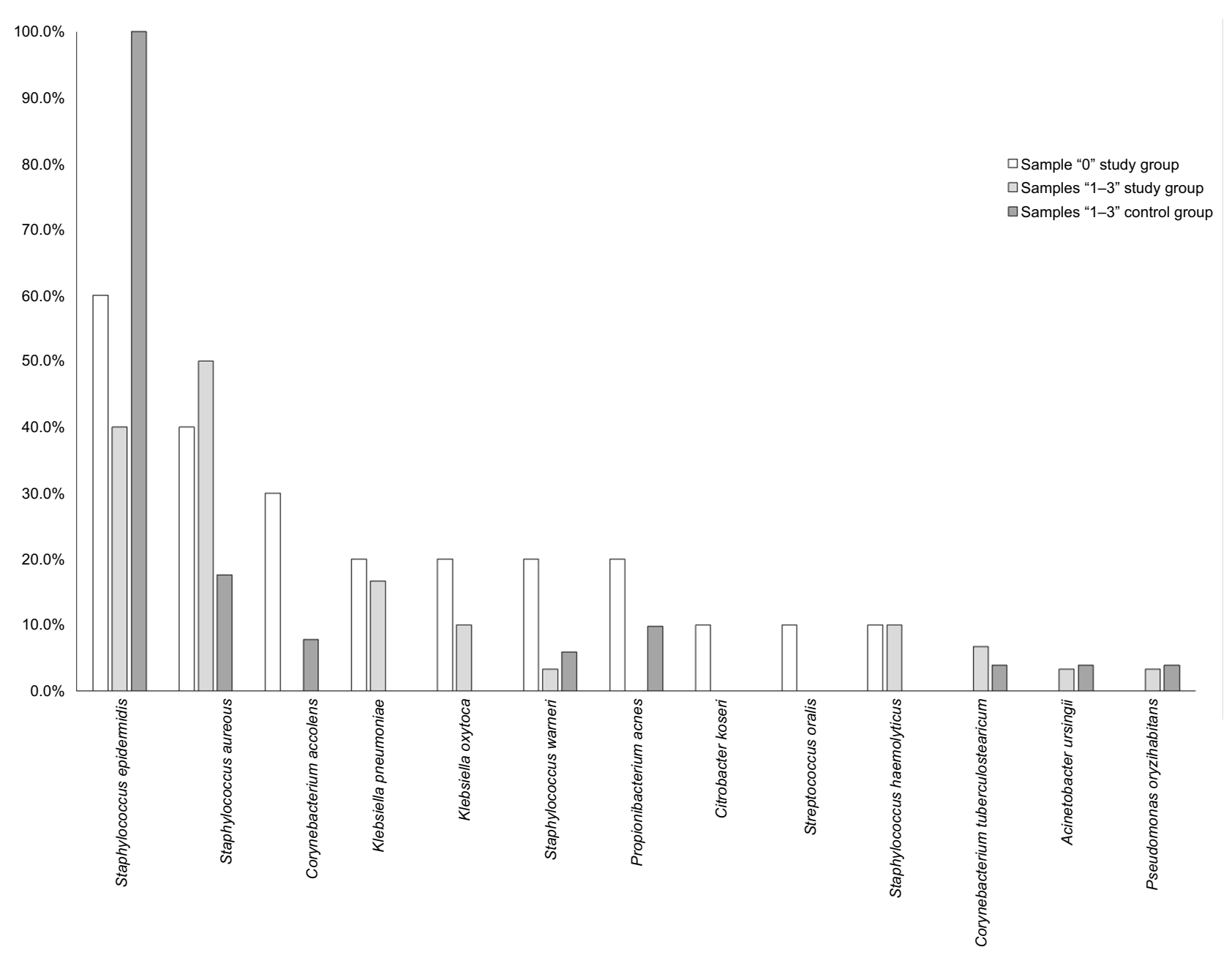

Figure I Qualitative composition of the nasal flora.

infection and its composition after the complication was cured. Individuals who did not experience any related complications within at least 24 months of the first treatment of cross-linked HA qualified for the control group. Cultures made from nasal swabs were taken three times at 4-8 week intervals to determine the qualitative composition of the bacterial flora and the carriers of S. aureus and other species of bacteria. A significantly higher incidence of S. epidermidis was found in the control group, as well as a significantly lower incidence of $S$. aureus. Gram-negative rods of the family Enterobacteriaceae belonging to the Klebsiella spp. and Proteus spp. were only present in samples from the study group. A significantly higher incidence of $S$. aureus as a persistent component of the bacterial flora in the study group was found in comparison to the control group ( $40 \%$ vs $5.9 \%$ ). LBI was present at each cross-linked HA gel augmentation site in two out of the three $S$. aureus nasal carriers. S. aureus nasal carriage is an endogenous reservoir for various $S$. aureus infections, which often arise as complications from procedures. ${ }^{19,20}$ Statistical differences $(P=0.000)$ in the presence of $S$. epidermidis in the bacterial flora were significant when the control group was compared with the study group. Every individual in the control group was a persistent carrier of S. epidermidis, confirmed by the three subsequent samples taken. However, throughout the 40 samples taken from the study group, S. epidermidis was cultured from only $16(40 \%)$. Only three individuals were considered persistent carriers of this species. Shruti Naik et al presented the results of studies that defined a mechanism for mutual interactions of cellular mediators and how the commensal microflora influences the immunity of the skin. Colonization by $S$. epidermidis in particular induces a congenital immune response related to the production of IL-17A by TCD8 ${ }^{+}$, which in this context is not an inflammatory reaction, but rather the expression of an evolutionarily developed defense mechanism preventing the invasion of various pathogens. ${ }^{21}$ Furthermore, Iwase et al proved that clinical isolates of $S$. epidermidis are capable of stopping the formation of biofilms by $S$. aureus through the production of serine protease, which contributes to antimicrobial function. The introduction of $S$. epidermidis into the nostrils of volunteers who were carriers of $S$. aureus caused a lowering of the rate of colonization by $S$. aureus. ${ }^{22}$ 
S. epidermidis also had the ability to stop $S$. aureus infection in individuals with unresolved atopic dermatitis changes. However, the link between these two microorganisms in skin affected by disease remains unclear. ${ }^{17}$ At this stage of research, we are unable to answer the question of whether the growth of bacteria was the etiological factor of infection. However, it is very likely that skin disturbed in its normal flora composition does not give full protection against the invasion of pathogens and contributes to the infection. No one knows why some patients develop this complication and how this can be prevented. The problem of antibiotic therapy for LBI, probable BBI, is far beyond the scope of this article and is the subject of our further research.

\section{Conclusion}

The results of this study confirm the hypothesis that $S$. epidermidis provides a protective function guarding the skin from LBI, probable BBI. S. epidermidis and other coagulase-negative staphylococci are considered to be the main representatives of physiological flora of the skin. Other commensal microorganisms in the flora include bacteria of the genera Corynebacterium, Propionibacterium, Brevibacterium, and Micrococcus. ${ }^{16}$ However, the study group was characterized by the presence of bacteria typically considered as pathogens. Gram-negative bacteria, with the exception of Acinetobacter spp., generally are not isolated from the skin, but are considered to be contamination originating from the bowel. ${ }^{16}$ We found a significantly higher incidence of Gramnegative rods of the family Enterobacteriaceae in samples taken from the study group compared to the control group (40\% vs 3.9\%). Significant abnormalities in the qualitative composition of the nasal flora of patients with LBI, probable $\mathrm{BBI}$, at the site of cross-linked HA gel augmentation were found. These abnormalities consisted of a significantly lower incidence of $S$. epidermidis, and more common incidences of the $S$. aureus carrier state in the study group, compared to the control group. The persistence of Gram-negative rods of the family Enterobacteriaceae in the nostrils is an interesting discovery, especially based on the case of Klebsiella spp. ${ }^{23-25}$ According to our knowledge, the question remains open regarding the influence of antibiotic therapy on the composition of the physiological skin flora of healthy individuals. ${ }^{16}$ This fact is of crucial importance to the development of procedural algorithms aiming to reduce the risk of complications after treatments using cross-linked HA gel, especially if new complications after HA gel augmentation might still occur. ${ }^{26}$ All our patients were successfully cured with the use of antibiotics and hyaluronidase.
There are questions that remain to be answered. Are there any advantages in using antibacterial compounds to eradicate the permanent carriage of $S$. aureus or other potentially pathogenic bacteria, which may be a subsequent cause of even more severe problems? Should prophylactic antibiotic therapy be given to individuals carrying $S$. aureus? Would the proper use of antiseptics while performing the HA augmentation procedure prevent the occurrence of LBI, probable BBI? Should a cream containing a specific strain of $S$. epidermidis be used prophylactically at the planned site of the procedure a few days before cross-linked HA gel augmentation? Further research in this area is required to answer these questions.

In conclusion, 1) the contents of bacterial flora on the skin differed in patients with LBI than in the control group; 2) the control group bacterial skin flora was dominated by S. epidermidis; and 3) patients with LBI had a bacterial skin flora dominated by potentially pathogenic bacteria.

\section{Author contributions}

IN supervised, designed, and coordinated the study, collected data and samples, performed microbiological testing and data analysis, and drafted the article. WM supervised, designed, and coordinated the study, enrolled the patients and treated them, collected the data and samples, performed data analysis, drafted the article, and funded the study. RO designed and coordinated the study, enrolled the patients and treated them, collected the data and samples, performed data analysis, drafted the article, and funded the study. KS enrolled the patients and treated them, collected the data and samples, participated in data analysis, and funded the study. AK-U enrolled the patients and treated them, collected the data and samples, participated in data analysis, and funded the study. ES-K performed data collection and microbiological testing, and participated in data analysis. JS participated in data analysis and performed statistical analysis. ZS participated in data analysis, drafted the article, and translated it into English. GM supervised the study, participated in data analysis, and drafted the article. All authors contributed toward data analysis, drafting and revising the paper and agree to be accountable for all aspects of the work.

\section{Disclosure}

The authors report no conflicts of interest in this work.

\section{References}

1. Sathianathan M, Hegde A, Vickery K, Deva AK. The role of biofilm in hyaluronic acid filler: an in vitro study. Plast Reconstr Surg 2013;132(4S-1):100-101. 
2. Grippaudo FR, Pacilio M, Di Girolamo M, Dierckx RA, Signore A. Radiolabelled white blood cell scintigraphy in the work-up of dermal filler complications. Eur J Nucl Med Mol Imaging. 2013;40(3): 418-425.

3. Heppt M, Hartmann D, Reinholz M, Feller-Heppt G, Ruzicka T, Gauglitz GG. [Fillers and associated side effects]. HNO. 2015;63(7):472-480. German.

4. Funt D, Pavicic T. Dermal fillers in aesthetics: an overview of adverse events and treatment approaches. Clin Cosmet Investig Dermatol. 2013;6:295-316.

5. De Boulle K, Heydenrych I. Patient factors influencing dermal filler complications: prevention, assessment, and treatment. Clin Cosmet Investig Dermatol. 2015;8:205-214.

6. Marusza W, Mlynarczyk G, Olszanski R, et al. Probable biofilm formation in the cheek as a complication of soft tissue filler resulting from improper endodontic treatment of tooth 16. Int J Nanomedicine. 2012;7:1441-1447.

7. Lee JM, Kim YJ. Foreign body granulomas after the use of dermal fillers: pathophysiology, clinical appearance, histologic features, and treatment. Arch Plast Surg. 2015;42(2):232-239.

8. Kim JH, Choi JS, Yun JH, et al. Foreign body reaction to injectable hyaluronic acid: late granuloma formation. Ann Dermatol. 2015;27(2):224-225.

9. Alhede M, Er O, Eickhardt S, et al. Bacterial biofilm formation and treatment in soft tissue fillers. Pathog Dis. 2014;70(3):339-346.

10. Dumitrascu DI, Georgescu AV. The management of biofilm formation after hyaluronic acid gel filler injections: a review. Clujul Med. 2013;86(3):192-195.

11. Neumeister B, Besenthal I, Liebich H. Klinikleitfaden Labordiagnostik [Laboratory Diagnostics]. Germany: Urban and Fischer; 2003:502. Polish.

12. Bjarnsholt T, Tolker-Nielsen T, Givskov M, Janssen M, Christensen LH. Detection of bacteria by fluorescence in situ hybridization in culturenegative soft tissue filler lesions. Dermatol Surg. 2009;35(Suppl 2): $1620-1624$.

13. Christensen L. Normal and pathologic tissue reactions of soft tissue gel fillers. Dermatol Surg. 2007;33(Suppl 2):5186-5172.
14. Bjarnsholt T. The role of bacterial biofilms in chronic infections. APMIS Suppl. 2013;136:1-51.

15. Wu H, Moser C, Wang HZ, Høiby N, Song ZJ. Strategies for combating bacterial biofilm infections. Int J Oral Sci. 2015;7(1):1-7.

16. Grice EA, Segre JA. The skin microbiome. Nat Rev Microbiol. 2011;9(4):244-253.

17. Sanford JA, Gallo RL. Functions of the skin microbiota in health and disease. Semin Immunol. 2013;25(5):370-377.

18. Frank DN, Feazel LM, Bessesen MT, Price CS, Janoff EN, Pace NR. The human nasal microbiota and Staphylococcus aureus carriage. PLoS One. 2010;5(5):e10598.

19. Eiff CH, Becker K, Machka K, Stammer H, Peters G. Nasal carriage as a source of Staphylococcus aureus bacteremia. NEngl J Med. 2001;344(1): $11-16$.

20. Heiman FL, Melles WDC, Vos MC, et al. The role of nasal carriage in Staphylococcus aureus infections. Lancet Infect Dis. 2005;5(12):751-762.

21. Shruti Naik S, Bouladoux N, Linehan JL, et al. Commensal-dendriticcell interaction specifies a unique protective skin immune signature. Nature. 2015;520(7545):104-108.

22. Iwase T, Uehara Y, Shinji H, et al. Staphylococcus epidermidis Esp inhibits Staphylococcus aureus biofilm formation and nasal colonization. Nature. 2010;465(7296):346-349.

23. Dethlefsen L, Relman DA. Incomplete recovery and individualized responses of the human distal gut microbiota to repeated antibiotic perturbation. Proc Natl Acad Sci U S A. 2011;108(Suppl 1): 4554-4561.

24. Antonopoulos DA, Huse SM, Morrison HG, SchmidtTM, Sogin ML, Young VB. Reproducible community dynamics of the gastrointestinal microbiota following antibiotic perturbation. Infect Immun. 2009;77(6):2367-2375.

25. Dethlefsen L, Huse S, Sogin ML, Relman DA. The pervasive effects of an antibiotic on the human gut microbiota, as revealed by deep $16 \mathrm{~S}$ rRNA sequencing. PLoS Biol. 2008;6(11):e280.

26. Signorini M, Liew S, Sundaram H, et al. Global aesthetics consensus: avoidance and management of complications from hyaluronic acid fillers - evidence- and opinion-based review and consensus recommendations. Plast Reconstr Surg. 2016;137(6):961e-971e.
Infection and Drug Resistance

\section{Publish your work in this journal}

Infection and Drug Resistance is an international, peer-reviewed openaccess journal that focuses on the optimal treatment of infection (bacterial, fungal and viral) and the development and institution of preventive strategies to minimize the development and spread of resistance. The journal is specifically concerned with the epidemiology of antibiotic

\section{Dovepress}

resistance and the mechanisms of resistance development and diffusion in both hospitals and the community. The manuscript management system is completely online and includes a very quick and fair peerreview system, which is all easy to use. Visit http://www.dovepress.com/ testimonials.php to read real quotes from published authors. 